\title{
Development of a regenerated Beryllium target and a thermal test facility for Compact Accelerator-based Neutron Sources
}

\author{
Jean-François Muraz ${ }^{l}$, Daniel Santos ${ }^{I}$, Véronique Ghettal, Julien Giraud ${ }^{1}$, Julien Marpaud ${ }^{l}$, Marine Hervél, Pascal \\ Sortais $^{2}$, Mauro Forlino ${ }^{3}$
}

\author{
${ }^{1}$ Laboratoire de Physique Subatomique et de Cosmologie 38026 Grenoble, France \\ ${ }^{2}$ Polygon Physics 38000 Grenoble \\ ${ }^{3}$ ARFITEC internship engineer
}

\begin{abstract}
Recently, the possibility to use compact accelerators coupled to high current ion sources for the production of intense low energy proton or deuteron beams has motivated many research laboratories to develop accelerator based neutrons sources for several purposes, including Neutron Capture Therapy (NCT). The NCT needs a high flux, about $10^{9} \mathrm{n} \cdot \mathrm{cm}^{-2} \cdot \mathrm{s}^{-1}$, of thermal neutrons $(\mathrm{E}<10 \mathrm{keV})$ at the tumour site. Up to now, the NCT required neutron flux was mainly delivered by nuclear reactors. However, the production of such neutron flux is now possible using proton or deuteron beams on specific targets able to stand a high pow er $(\sim 15 \cdot 30 \mathrm{~kW})$ on a small area $\left(\sim 10 \mathrm{~cm}^{2}\right)$. This specific target design, materials and supports, has to cope with extreme physical constraints . The LPSC team has conceived an original solution formed by a thin $(8 \mu \mathrm{m})$ rotating beryllium target depos ited on a graphite wheel and coupled with a beryllium sputtering device for periodic ${ }^{9} \mathrm{Be}$ layer restoration. By means of ${ }^{9} \mathrm{Be}(\mathrm{d}, \mathrm{n}){ }^{10} \mathrm{~B}$ nuclear reaction, this target irradiated by a $10 \cdots 20 \mathrm{~mA}$ deuteron beam $(1.45 \mathrm{MeV})$ should produce the required neutron flux. In order to validate the target design of the neutron flux production and the beryllium target thermal capabilities, we built a $30 \mathrm{~cm}$ diameter rotating Beryllium target prototype and a compact electron beam line able to deliver a power density of $3 \mathrm{~kW} / \mathrm{cm}^{2}$.
\end{abstract}

\section{Context}

Neutron sources are used in many domains such as isotopes production, non-destructive imaging, subsurface exploration, silicon transmutation doping, and, in the medical, domain Neutron Capture Therapies profiting of the huge ${ }^{10} \mathrm{~B}$ neutron capture cross section [1-2] producing an energetic alpha particle and $\mathrm{Li}$ nuclear recoil with a $478 \mathrm{keV}$ gamma ray in $94 \%$ of cases [fig 1]

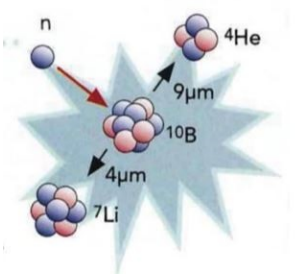

Fig. 1. Boron Neutron Capture nuclear reaction

Unlike nuclear reactors, accelerator-based neutron sources appear like safe, compact and cheap solutions to produce a high neutron flux [3]. This is especially true for Neutron Capture Therapies, which require the highest thermal neutron flux produced by the smallest compact neutron source suitable to sit in a hospital.

In this context, the LPSC team realized that one of the critical points for compact accelerator-based neutron source developments is the robustness of the target under a continuous high current beam.

\section{The regenerated Beryllium rotating target}

The LPSC team has developed an original beryllium rotating target where the beryllium layer can be regenerated. This target has been designed to cope with a power density of $3 \mathrm{~kW} / \mathrm{cm}^{2}$.

In a square aluminium vacuum chamber, a $15 \mathrm{~mm}$ thick circular graphite disc is turning at 1 to $3 \mathrm{~Hz}$ [fig 2]. This rotation is produced and controlled by a stepping motor and the rotation torque is transferred to the disc shaft using a magnetic shaft coupling to avoid rotary seals and so to reduce the target maintenance.

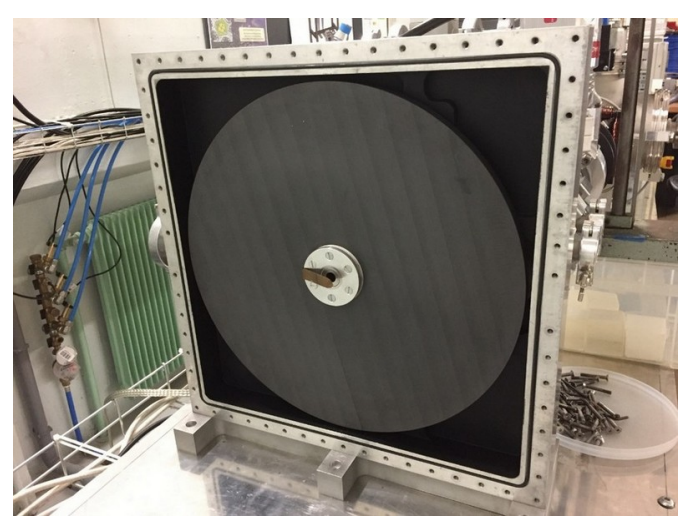

Fig. 2. Internal view of the beryllium target

\footnotetext{
* Corresponding author: muraz@1psc.in2p3.fr
} 
Fig. 2. Internal view of the beryllium target

The beryllium layer is deposited and regenerated on the graphite disc by Ion Beam Sputtering [fig 3]. This process consists of using a compact ECR ion source to produce a specific beam which hits a pure beryllium target oriented in such a way that the beryllium atoms are sputtered into the graphite surface.

\section{CONVENTIONAL IBS}

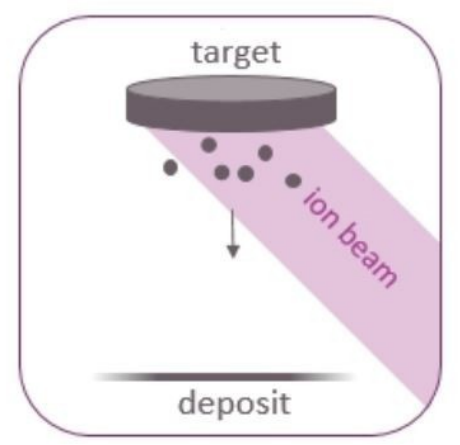

Fig. 3. Ion Beam Sputtering working principle

The heat, produced by the beam on the beryllium layer and the graphite disc, is transferred by radiation to the aluminium vacuum chamber, which is water-cooled on both sides [fig 4].

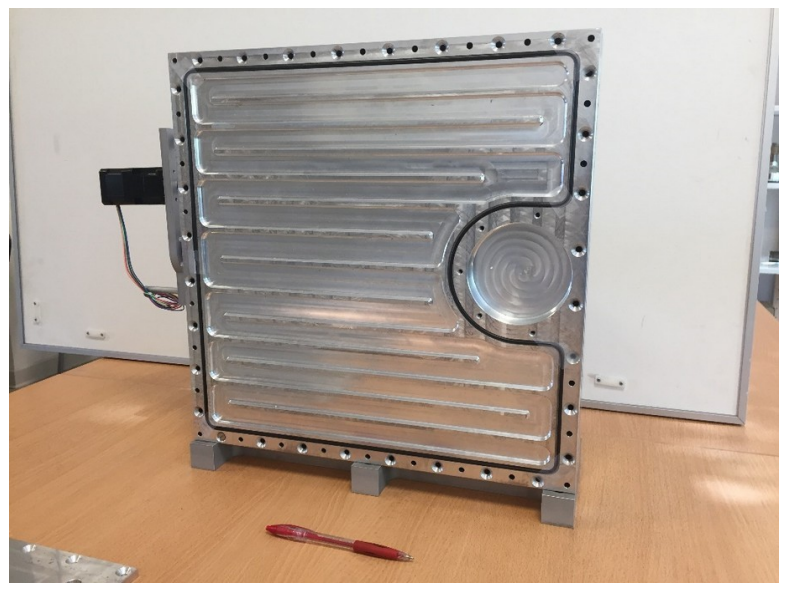

Fig. 4. Water cooling channels

The graphite disc temperature is measured by 2 optical pyrometers placed before and after the beam spot.

\section{The electron beam thermal test facility}

In order to test in situ our target developments, the LPSC team has developed a thermal test facility [fig 5].

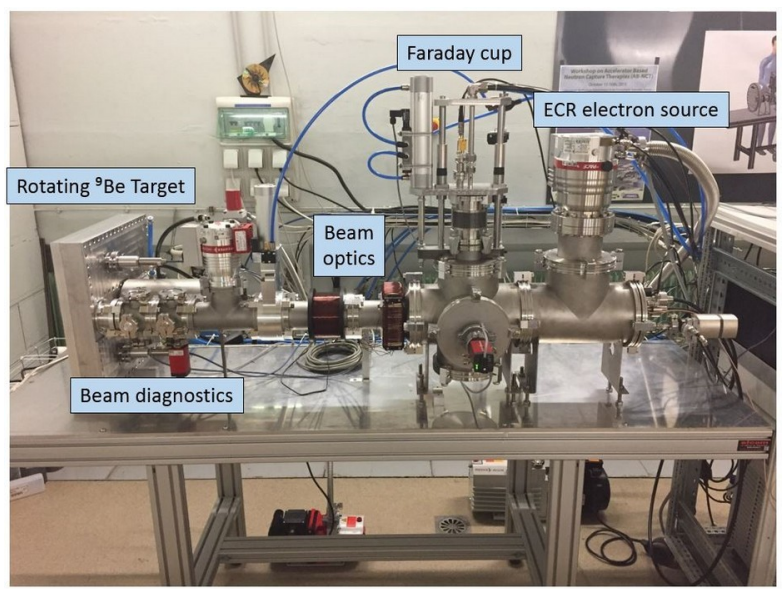

Fig. 5. The $3 \mathrm{~kW}$ thermal electron beam facility

This facility is based on an ECR electron source produced by the Polygon Physics Company. The ECR electron source [fig 6] is set to produce a $18 \mathrm{keV}-168$ $\mathrm{mA}$ electron beam.

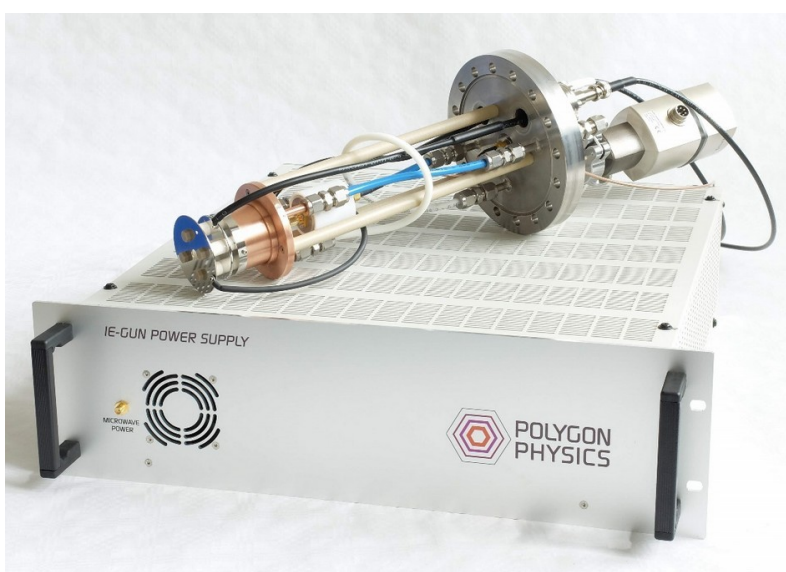

Fig. 6. The ECR electron source

The beam current is measured by an extractible Faraday cup, which is water-cooled [fig 7].

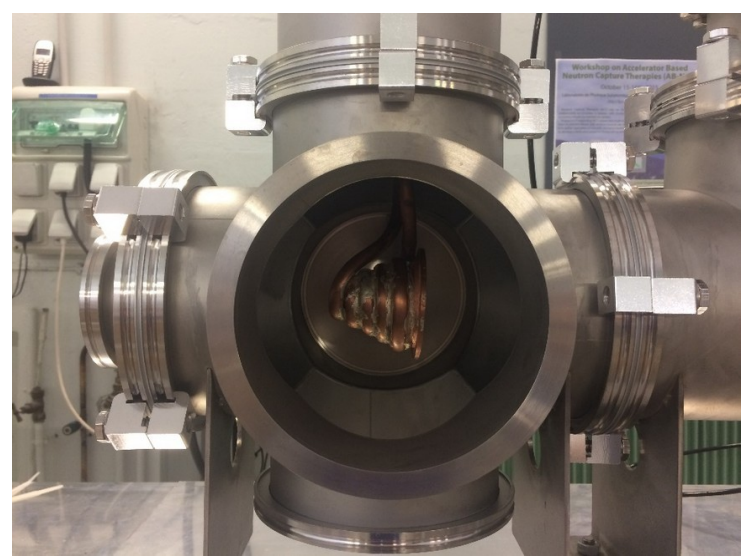

Fig. 7. The Faraday cup 
The beam can be positioned using two magnetic steerers and focalized by acting on a magnetic solenoid [fig 8].

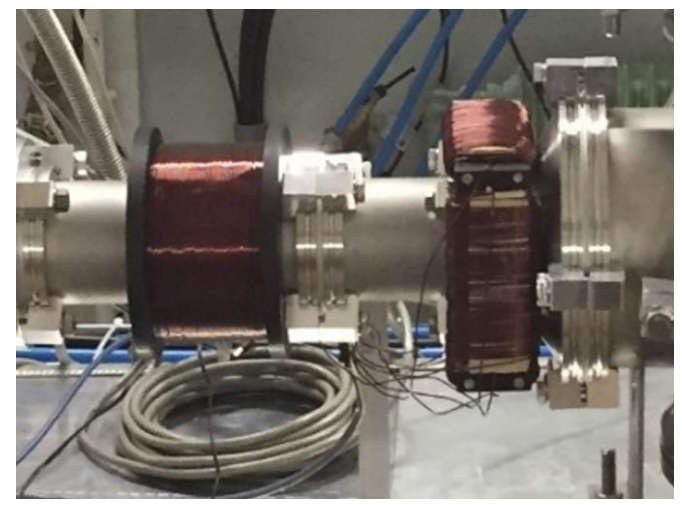

Fig. 8. The electron beam optics

Due to its high intensity, the electron beam produces fluorescence light in the visible range in the residual gas contained in the beam pipes. The beam size and position are then controlled online by a nonintersecting Beam Induced Florescence diagnostic device. Two perpendicular fast cameras will do the transverse profile measurement.

\section{First measurements}

We performed very preliminary measurements to check the target behaviour under power variation [fig 9]. These measurements were done up to $2.5 \mathrm{~kW} . \mathrm{cm}^{-2}$.

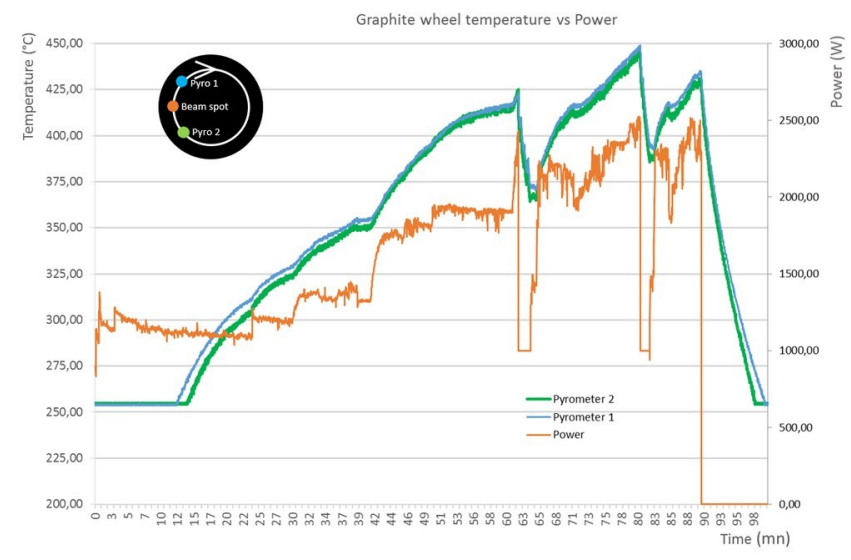

Fig. 9. Target thermal behaviour according to beam power

According to these preliminary measurements, we can clearly see the fast thermal response of the target under the beam power solicitations.

\section{Conclusion}

We have designed and built a regenerated rotating beryllium target and an electron beam facility to perform thermal target tests up to $3 \mathrm{~kW} \cdot \mathrm{cm}^{-2}$. We performed some preliminary measurements up to $2.5 \mathrm{~kW} . \mathrm{cm}^{-2}$ confirming the good thermal abilities of the target design. Our next goals are to complete the thermal tests and to study the ${ }^{9} \mathrm{Be}$ layer behaviour under the 3 $\mathrm{kW} \cdot \mathrm{cm}^{-2}$ power density as a function of working time. An NIM article on all these developments is being written and will be soon published.

\section{References}

[1] M.E.Capoulat, N. Sauzet, A.A. Valda, L. Gagetti, O. Guillaudin, L. Lebreton, D. Maire, P. Mastinu, J. Praena, Q. Riffard, B. Tampon, D. Santos, A.J Kreiner Neutron spectrometry of the ${ }^{9} \mathrm{Be}(\mathrm{d}$ $(1.45 \mathrm{MeV}), n)^{10} B$ reaction for accelerator-based BNCT https://doi.org/10.1016/j.nimb.2019.03.005

[2] M.E. Capoulat, D.M. Minsky, A.J. Kreiner, Computational assessment of deep-seated tumor treatment capability of the $9 \mathrm{Be}(d, n) 10 B$ reaction for accelerator-based Boron Neutron Capture Therapy (AB-BNCT), Phys. Medica. 30 (2014), https://doi.org/10.1016/j.ejmp.2013.07.001.

[3] T.E. Blue, J.C. Yanch, Accelerator-based epithermal neutron sources for boron neutron capture therapy of brain tumors, J. Neurooncol. 62 (2003) https://doi.org/10.1023/A:1023247222043 